\title{
PARAMETER OPTIMIZATION FOR ELECTRON BEAM MELTING OF IN718 BASED ON MELT POOL CHARACTERIZATION
}

\author{
Xiao Ding ${ }^{1}$,Yuichiro Koizumi ${ }^{2}$, Akihiko Chiba $^{2}$ \\ ${ }^{1}$ Department of Materials Processing, Graduate School of Engineering, Tohoku University, 6-6-11, Aramaki, Aoba-ku, Sendai 980-8579, \\ Japan \\ ${ }^{2}$ Institute for Materials Research (IMR), Tohoku University, 2-1-1 Katahira, Aoba-ku, Sendai 980-8577, Japan
}

Keywords: process parameter, melt pool, electron beam melting, IN718

\begin{abstract}
Single bead experiments were conducted to clarify the relationship between process parameters and melt pool geometry based on melt pool characterization, aiming to obtain a better understanding of Electron Beam Melting (EBM) additive manufacturing. INCONEL ${ }^{\circledR} 1718$ polycrystalline plate and gas atomization powders were used to generate melt pool geometries by various beam power and scan speed combinations. The surface morphology and cross section microstructures were analyzed. The surface morphology indicates that input energy play an important role in single beads continuity. The microstructure of fully columnar grains is verified by cross-section profile of single beads. Furthermore, the melt pool widths and depth increase almost linearly with beam power on the basis of the cross-sectional geometrical profiles. A reciprocal relationship exists between width/depth ratio and line energy. Finally, process condition has profound effect on crystal growth and grain structure. Grains grow along different direction in relatively high line energy cases. However, grains grow almost along the same direction namely grow vertically in relatively low line energy cases.
\end{abstract}

\section{Introduction}

Electron beam melting, as a powder bed based additive manufacturing technology, is used to produce metallic structures via layer by layer melting with an electron beam. Due to its amazing advantages such as high level of geometric freedom together with excellent material properties, EBM has attracted increasing attention within automotive and aerospace industry [1, 2]. Compared with selective laser melting (SLM), EBM provides higher energy density, higher scan speed and higher energy absorption coefficient.

Although these advantages make EBM an extremely promising additive manufacturing method, there are many challenges that need to be overcome to obtain a better understanding of EBM process. EBM process is considerably complex, involving many physical phenomena such as melting and solidification of the melt pool, diffusive and convective heat conduction in the melt pool, capillary effect, radiation and gravity, etc [3]. During EBM process, thermal history and temperature distribution are very intricate including the occurrence of ultrahigh temperature gradients and cooling rates, resulting from localized high intensity heat input [4]. The temperature gradients and cooling rates control the formation, motion, and solidification rate of the melt pool, determining the microstructures and mechanical properties.

Previous researchers have proven that microstructures can be controlled by optimization of process parameters (such as power, scan speed, hatch spacing, layer thickness, scan pattern, etc.) $[5,6]$.

\footnotetext{
${ }^{1}$ INCONEL is a registered trademark of Special Metals
}

Gockel et al [7] observed columnar grains at different power and velocity, with more equiaxed grains present as the power was increased and the area remained constant. Helmer et al [8] studied the effect of beam focus on grain structures, indicating that defocused beam results in a more prefect columnar structure while stray grains appear with focused beam. Korner et al [9] found that the grain structure becomes equiaxed from columnar with the same area energy but lower line offset and higher scan speed. In order to obtain the desired microstructures, the effect of process parameters on microstructures needs to be investigated.

The process parameters, especially power and speed, have profound influence on melt pool geometry, and melt pool geometry determines crystal growth and microstructure development. Therefore, in the present study, the effect of process parameters on melt pool geometry and microstructures were investigated by single bead experiments, which means that electron beam is scanned on base plate for only one single pass.

\section{Experiment Procedure}

An A2X EBM system (Arcam AB) was used to perform single bead experiments. The IN718 base plate was placed in the chamber of EBM system, keeping the surface horizontal as shown in Figure 1. Figure 2 shows the microstructure of IN718 base plate, presenting fully equiaxed grains. Figure 3 shows the result of single bead experiment. Single scans of electron beam with various combinations of power and scan speeds were conducted on IN718 base plate for the following three cases, Case A: with neither preheating nor powder, Case B: with preheating at $850{ }^{\circ} \mathrm{C}$ without power, and Case $\mathrm{C}$ : with preheat of $850{ }^{\circ} \mathrm{C}$ and with 5 layers of IN718 powders (layer thickness was $50 \mu \mathrm{m}$ ). The beam conditions were same for all the cases. The powers and scan speeds employed are listed in Table I.

The surface morphology was evaluated by confocal laser microscope to study the continuity of the single beads. The single beads were sectioned through the center then polished to observe the cross section geometry features and microstructures of melt pool by scanning electron microscope equipped with electron backscattering diffraction (SEM-EBSD).

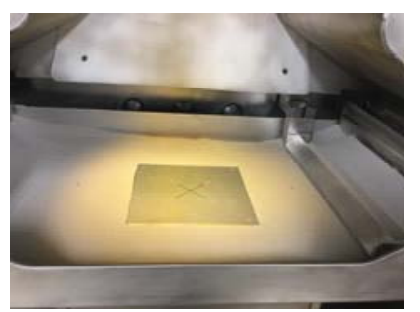

Figure 1. Position of base plate in EBM system 


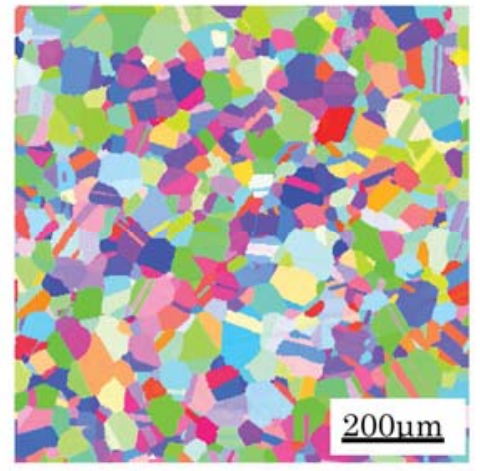

Figure 2. Microstructure of IN718 base plate



Figure 3. Result of single bead experiment

Table I. Electron-beam conditions for single bead experiments

\begin{tabular}{|c|c|}
\hline Process Parameter & \multicolumn{1}{|c|}{ Value } \\
\hline Beam Power(W) & $100,200,400,600,800,1000$ \\
Scan Speed(mm/s) & $100,300,1000,3000,10000$ \\
\hline
\end{tabular}

\section{Results and Discussions}

Surface morphology of single beads

The surface morphology of single beads provides valuable information about melt pool and continuity of scan tracks. Figure 4 shows the surface morphologies of single beads formed with various combinations of powers and scan speeds for case A. For the beam conditions with low line energy density (i.e. high velocity, low power), which are indicated with light gray background, beads are hardly recognized. As the energy density is elevated, beads become distinguishable as seen in the images with horizontal lines background but a lot of cracks were formed. For single beads that were scanned by high energy density, the single beads are continuous as in the images indicated with oblique and vertical lines backgrounds. In the images with vertical lines background, the single beads are very smooth; however, for the oblique lines ones, both sides of melt pool were sunken.

For Case B, the base plate was preheated to $850^{\circ} \mathrm{C}$ before single bead experiment. Figure 5 shows the surface morphologies of single beads formed with various combinations of powers and scan speeds for case B. The surface morphology of Case B is similar to that of Case A. However, the light gray region was smaller because melt pool formed at lower line energy density in Case B. Furthermore, there are no cracks in horizontal lines region of Case $\mathrm{B}$ even though the single beads are not continuous. This is because preheat decreases temperature gradient, resulting in lower internal stress.

For Case C, a layer of powder was spread on the base plate and then was preheated to $850{ }^{\circ} \mathrm{C}$ before single bead experiment. This powder-spreading, preheat and single bead experiment process is repeated for the other 4 times. Figure 6 shows the surface morphologies of single beads formed with various combinations of powers and scan speeds for case B. The surface morphology of Case $\mathrm{C}$ is similar to that of Case $\mathrm{B}$. The continuity of melt pool in Case $\mathrm{C}$ is better than that of Case A and Case B. However, the horizontal lines region becomes larger because of balling effect resulting from powder layer, as shown by the arrows.

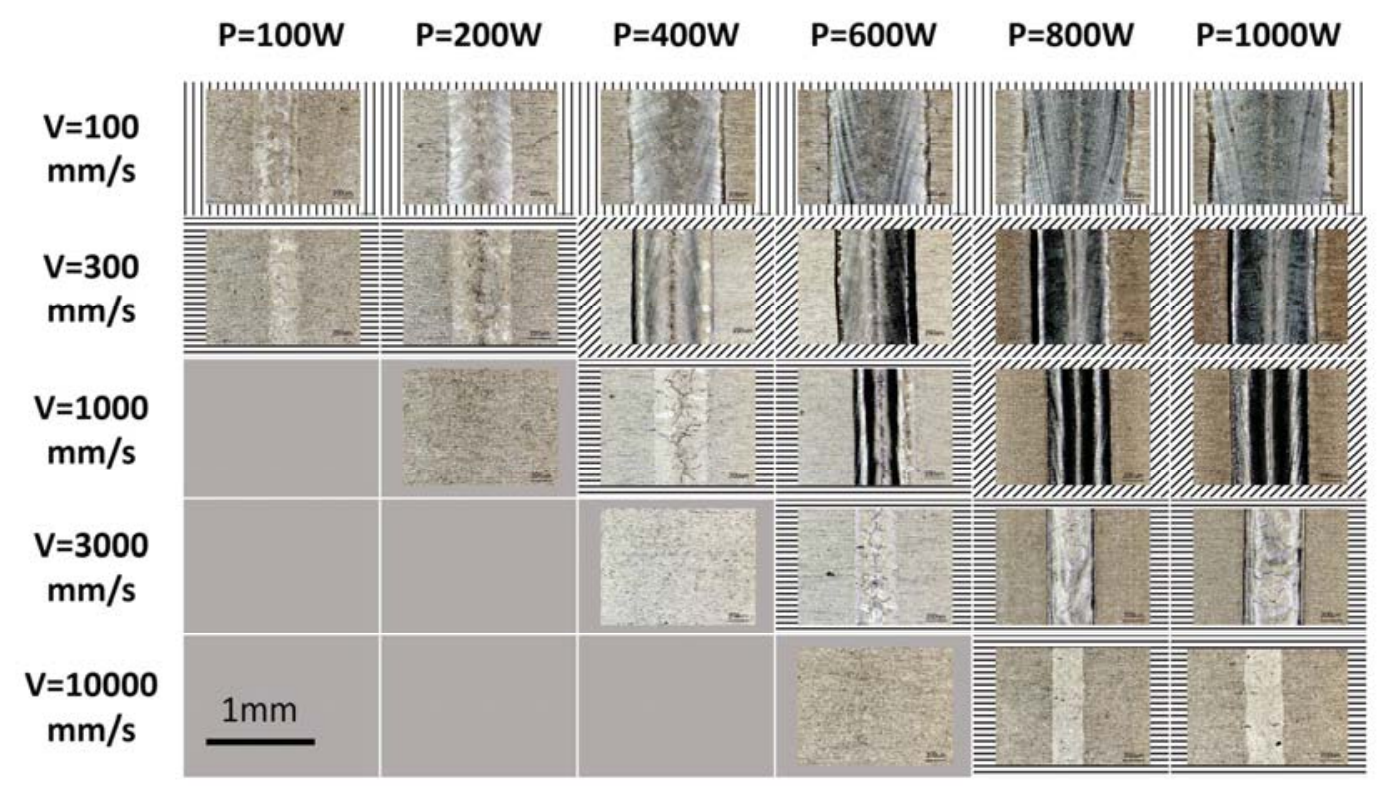

Figure 4. Laser microscope images of single beads of Case A showing melt pool with good quality (vertical and oblique lines region), melt pool with cracks (horizontal lines region) and no melt pool formed (light gray region). 




Figure 5. Laser microscope images of single beads of Case B showing melt pool with good quality (vertical and oblique lines region), continuous melt pool (horizontal lines region) and no melt pool formed (light gray region).

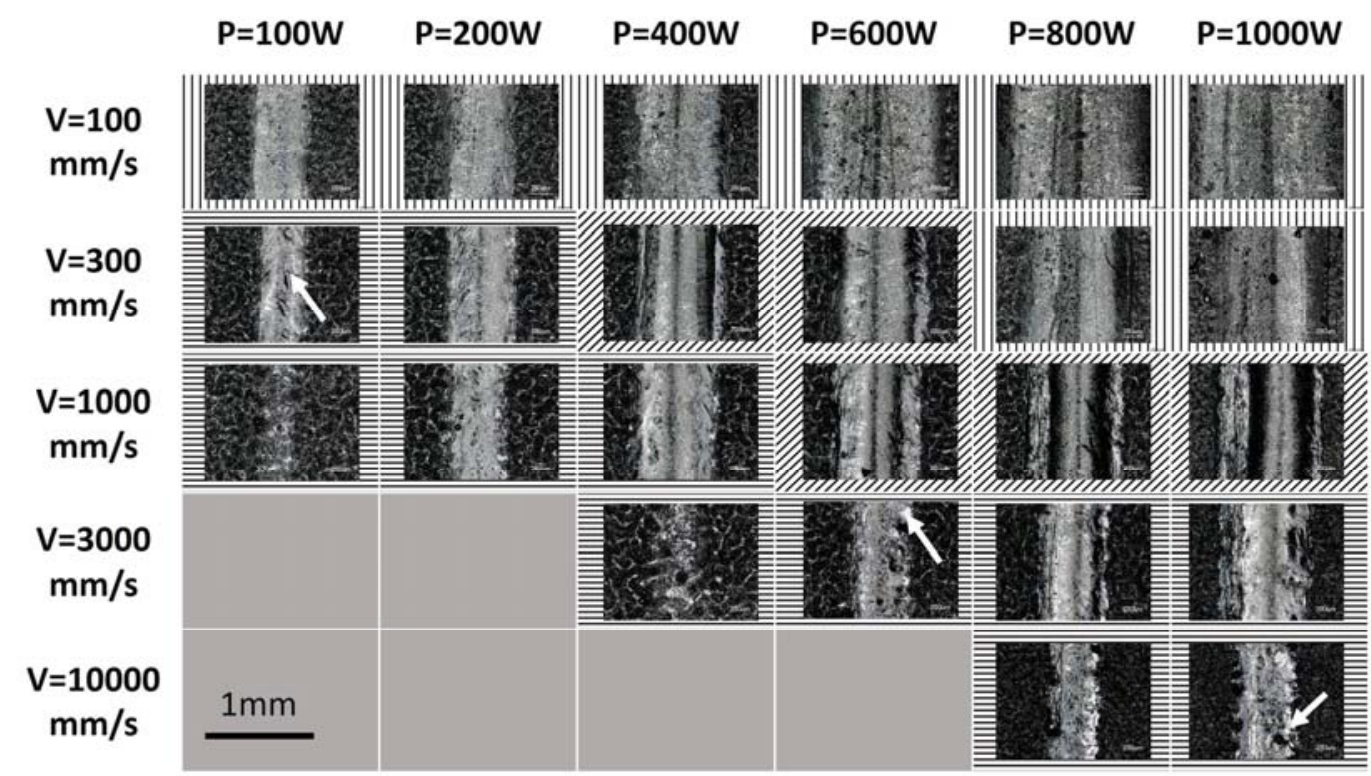

Figure 6. Laser microscope images of single beads of Case $\mathrm{C}$ showing melt pool with good quality (vertical and oblique lines region), melt pool with defects (horizontal lines region) and no melt pool formed (light gray region)

By comparative analysis, it has been revealed that preheating and existence of powder both have significant effects on the single bead's morphology. The width of melt pool with preheat is larger than that without preheat, and the width of melt pool with powder layers is larger than that without powder layers. In addition, the cracks that occurred in Case A where no preheat was performed were eliminated by preheating in Case B. This is because preheat decreases the temperature gradient around the melt pool, lowering the internal stress in the base plate. Preheat enlarged the region of melt pool with good quality (vertical and oblique lines region), and existence of powders enlarged the region of melt pool with defects (horizontal lines region). This means preheat makes single bead's continuity better while powders do the opposite.

Figure 7 shows the surface relief of single beads along the direction perpendicular to scan lines of different conditions for all three cases. With decreasing line energy (i.e. increasing beam scan speed or decreasing beam power), the height fluctuation becomes less significant. The height fluctuation of Case $\mathrm{C}$ is more pronounced than that of Case $\mathrm{B}$, and Case $\mathrm{B}$ is more significant than Case A. However, the difference of height evolution between three cases is so subtle that it can be ignored. 

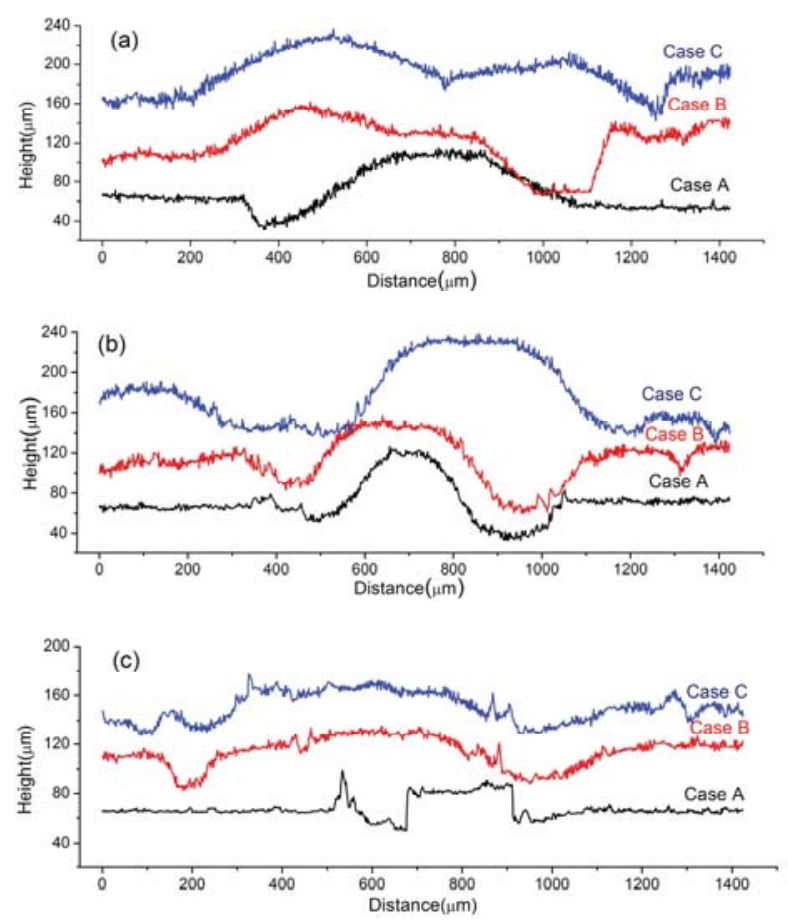

Figure 7. Surface relief of single beads along the direction that perpendicular to scan lines of (a) $\mathrm{P}=1000 \mathrm{~W}, \mathrm{v}=300 \mathrm{~mm} / \mathrm{s}$, (b) $\mathrm{P}=1000 \mathrm{~W}$, $\mathrm{v}=1000 \mathrm{~mm} / \mathrm{s}$, (c) $\mathrm{P}=600 \mathrm{~W}, \mathrm{v}=1000 \mathrm{~mm} / \mathrm{s}$.

Melt pool profiles of single beads

In order to further investigate melt pool morphology, all single beads were sectioned in the middle, perpendicularly to the scanning direction. Cross sections were then polished for metallography to show geometrical features and microstructures.

Figure 8 shows SEM cross-section images of single beads in Case A formed using multiple power and scan speed combinations. The melt pool is very easy to be distinguished from base plate. High power and low scan speed always resulted in larger melt pool and vice versa. With line energy increasing, the melt pool shape changes from elliptical to spherical then to conical shape. Fully columnar grains can be observed on cross-section for different process parameters. Furthermore, columnar grains grow along the direction perpendicular to interface of solid/liquid.

Figure 9 shows SEM cross-section images of single beads in Case B formed using multiple power and scan speed combinations. Compared with Case A, melt pool shape of Case B are similar but the size are larger.

Figure 10 shows SEM cross-section images of single beads in Case $\mathrm{C}$ formed using multiple power and scan speed combinations. For Case $\mathrm{C}$, the powders and base plate were melt together to form melt pool. Since the melt pool size is much larger than powder layer thickness, there is only little difference of melt pool geometry between Case B and Case C.

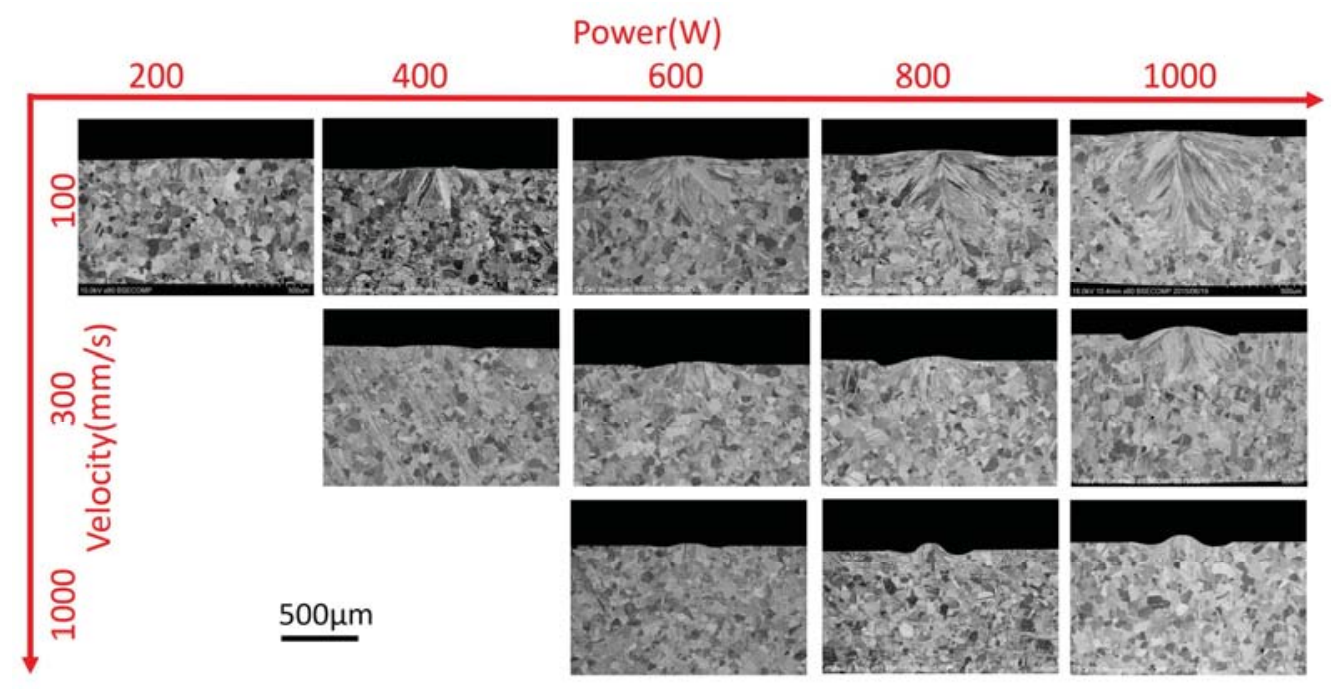

Figure 8. Cross-sectional SEM images of single beads for Case A 


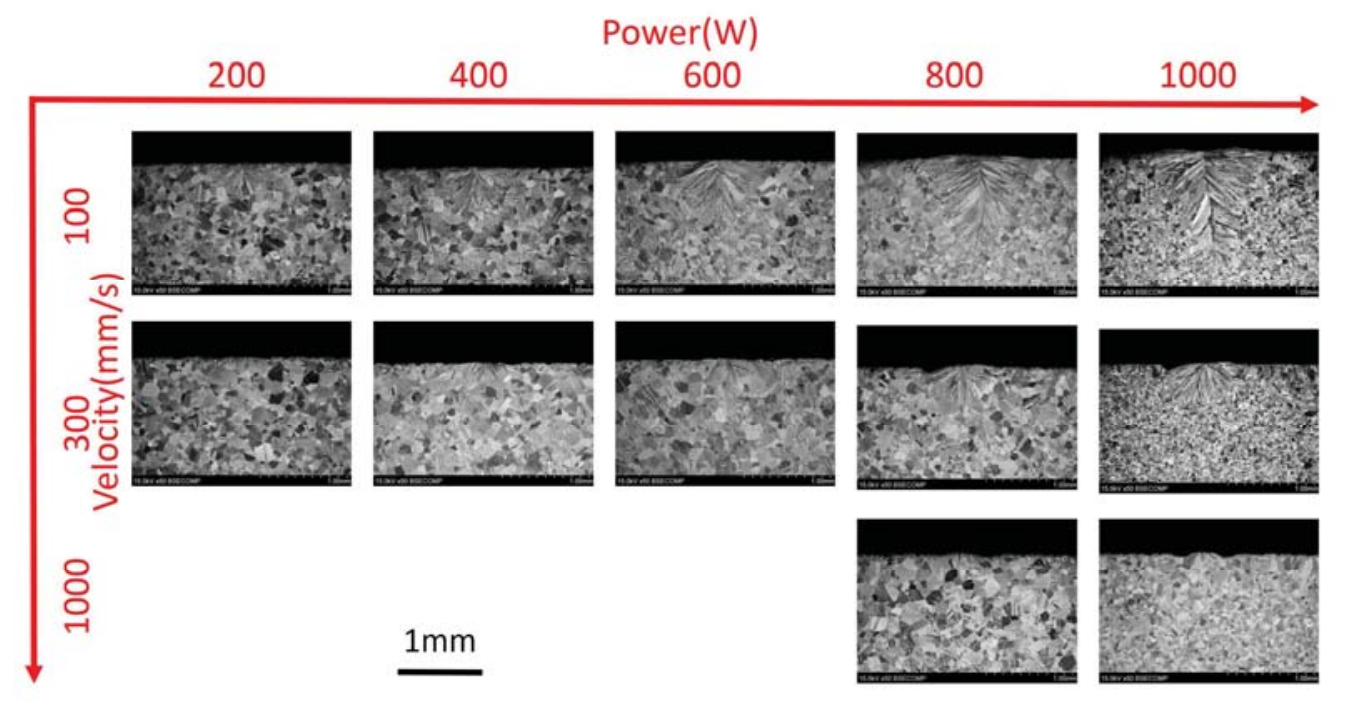

Figure 9. Cross-sectional SEM images of single beads for Case B

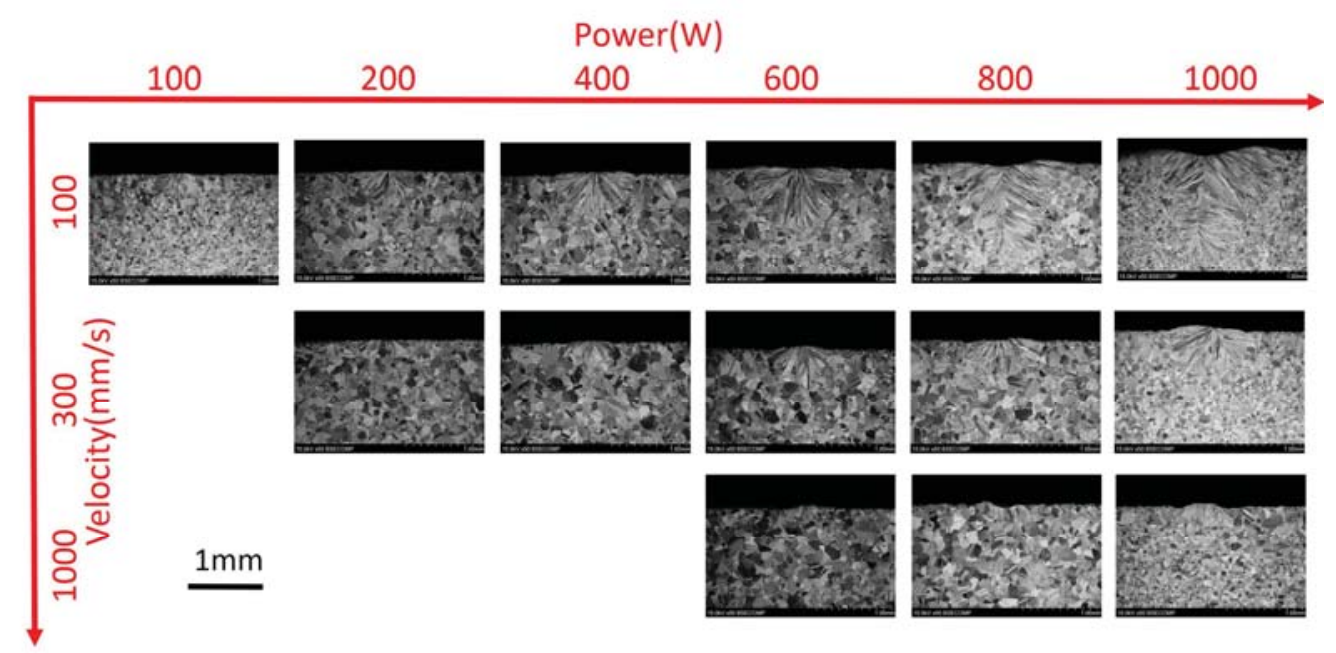

Figure 10. Cross-sectional SEM images of single beads for Case C

Figure 11 shows the melt pool widths and depth measured on the basis of the cross-sectional geometrical profiles for all the 3 cases. Width and depth of melt pool increases almost linearly with line energy increasing. Both preheat and existence of powder enlarge melt pool size. However, preheat has more profound effect on depth while powder layers affect width more significantly. Even when line energy is the same, melt pool sizes are not same. Larger power makes the melt pool wider and deeper when same line energy is performed. The widths and depth measured here can be used to choose appropriate line offset and layer thickness for experiment of simple structures for different process parameters. Preheat has more profound effect on depth, powder has more profound effect on width. A reciprocal relationship exists between width/depth ratio and line energy, as shown in Figure 12.

Figure 13 shows microstructures in transverse section of single beads for different conditions of all the 3 cases. The melt pool sizes of Case B and Case C are similar, and both are larger than that of Case A. For all conditions of 3 cases, fully columnar grain structures that grow perpendicular to solid/liquid surface were observed. For relatively high line energy cases, solid/liquid surfaces show half circle or inverted triangle shapes, and therefore grains grow along different direction since grains always grow perpendicular to solid/liquid surface. However, for relatively low line energy cases, melt pool is very shallow and shows half elliptical shape, which means the grains grow almost along the same direction, namely grow vertically. Epitaxial phenomenon occurs in many cases, as shown by black arrow. This is because large grain of base plate was not fully melted, and grain grows on the unmelted grain without new grain nucleation. It is very obvious that spacing of columnar grains is smaller when the base plate grains size is smaller. For polycrystalline base plate, it is difficult to control the average grain size and grain orientation. It is obvious that base plate grain structure also has significant effect on melt pool grain structure. Therefore, further detailed investigation about the effects of process conditions on crystal growth and grain structure is very difficult in the present study. Investigation using single crystal base plate is in progress. 

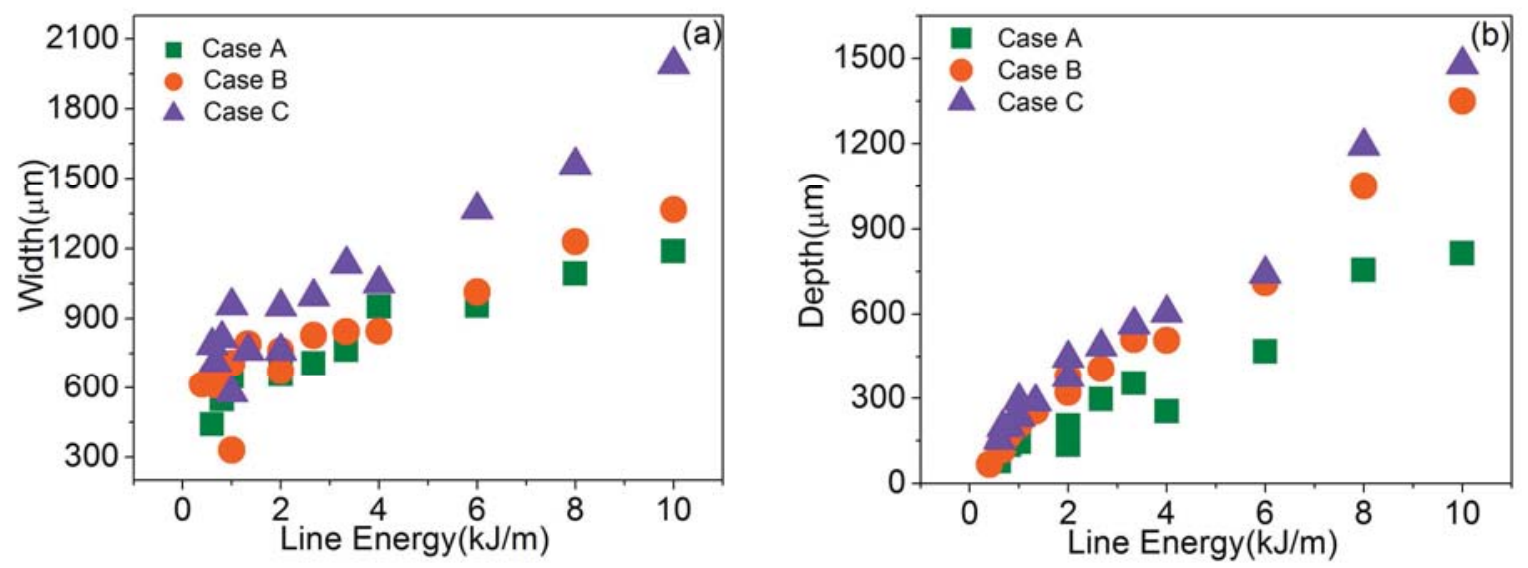

Figure 11. (a) Width and (b) depth of melt pool of different process conditions

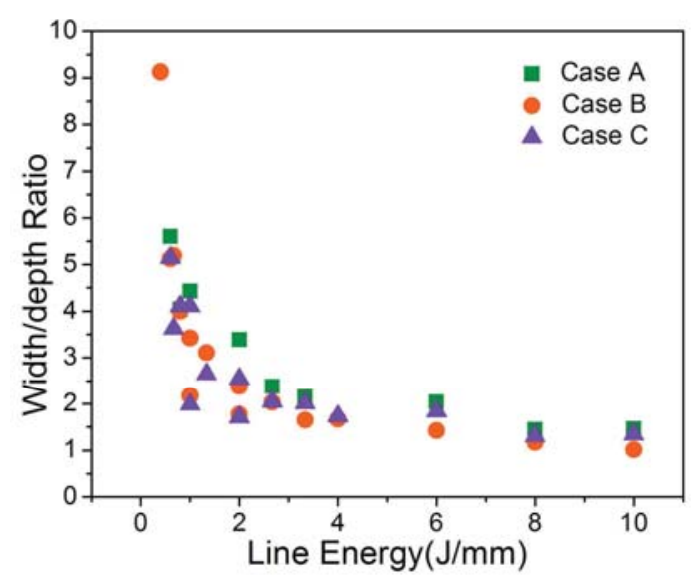

Figure 12. Relationship between line energy and width/depth ratio for 3 cases
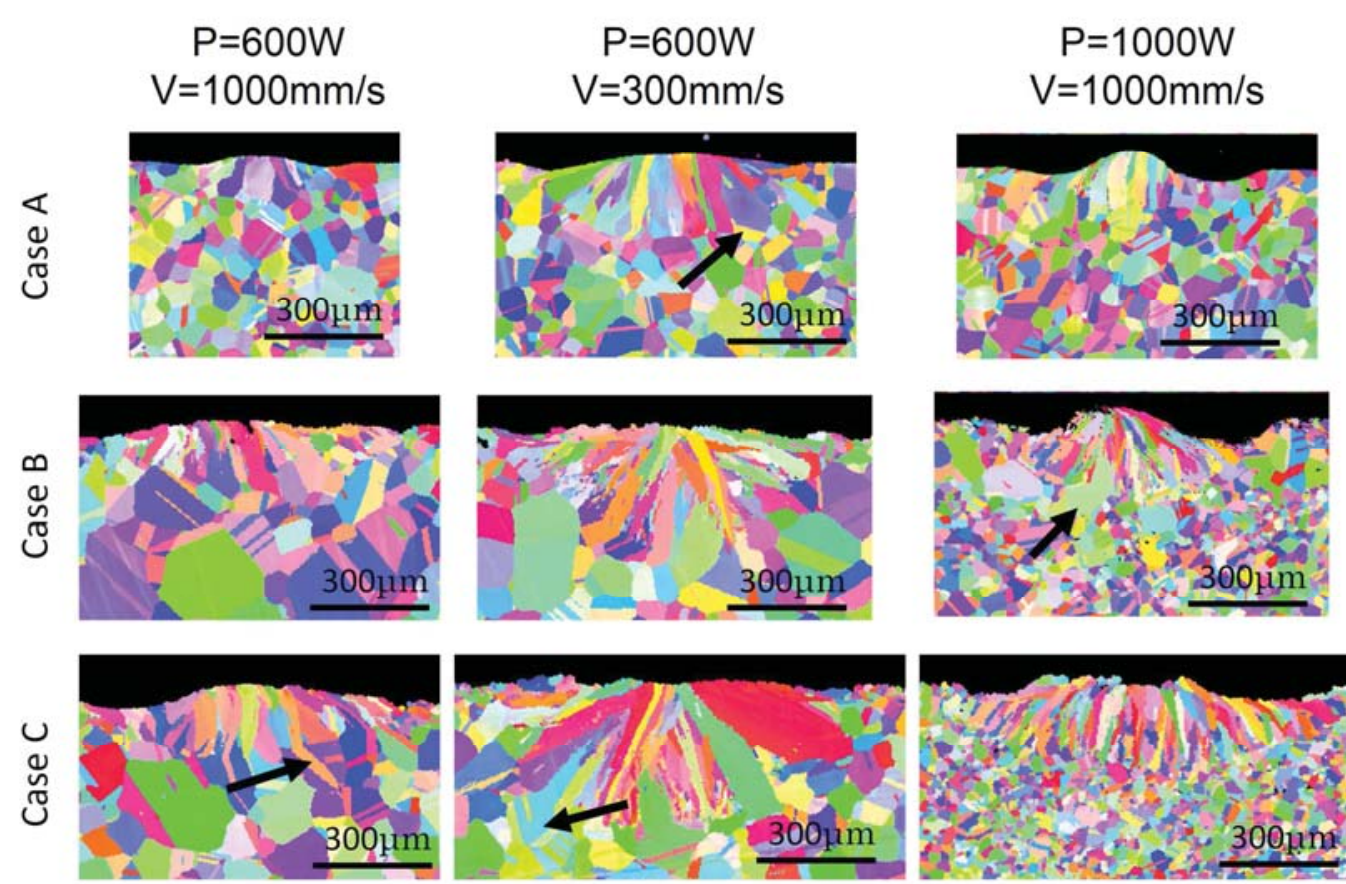

Figure 13. Microstructures of single beads of different conditions for 3 cases 


\section{Conclusions}

The single bead experiments for three cases with or without preheating and powder layers were carried out for parameter optimization based on melt pool characterization using the same parameter combinations. The surface morphology observations indicate that preheat and energy density play an important role in single beads continuity. With line energy increasing, the melt pool shape changes from elliptical to spherical then to conical shape. Finally, the melt pool widths and depth were measured as a function of beam power and scanning speed on the basis of the crosssectional geometrical profiles. It was found that a reciprocal relationship exists between width/depth ratio and line energy.

The microstructure of fully columnar grains, growing perpendicular to solid/liquid surface, is verified by cross-section profile of single beads. For relatively high line energy cases, grains grow along different directions; however, for relatively low line energy cases, grains grow almost along the same direction namely grow vertically. It can be concluded that process condition has profound effect on crystal growth and grain structure. However, further quantitative analysis is to be done with the absence of the effect of base plate. Experiments using single crystal base plate will be conducted to research the effect of melt pool geometry and grain orientation on crystal growth and grain structure development, in which the effect of grain size will be excluded.

\section{References}

1. S. Biamino et al., "Electron Beam Melting of Ti-48Al-2Cr-2Nb Alloy: Microstructure and Mechanical Properties Investigation," Intermetallics 19 (2011), 776-781.

2. L.E. Murr et al., "Microstructural Architecture, Microstructures, and Mechanical Properties for A Nickel-Base Superalloy
Fabricated by Electron Beam Melting," Metallurgical Materials Transactions A, 42A (2011), 3491-3508.

3. Carolin Körner, Elham Attar, and Peter Heinl, "Mesoscopic Simulation of Selective Beam Melting Processes," Journal of Materials Processing Technology, 211 (2011), 978-987

4. W. Yan et al., "Multiscale Modeling of Electron Beam and Substrate Interaction: A New Heat Source Model," Computational Mechanics, 56 (2015), 265-276.

5. S. Sun et al., "Build Direction Dependence of Microstructure and High-temperature Tensile Property of Co-Cr-Mo Alloy Fabricated by Electron Beam Melting," Acta Materialia, 64 (2014), 154-168.

6. S. Bontha et al., "Effects of Process Variables and Size-scale on Solidification Microstructure in Beam-based Fabrication of Bulky 3D Structures," Materials Science and Engineering A, 513-514 (2009), 311-318.

7. Joy Gockel, Jack Beuth, and Karen Taminger, "Integrated Control of Solidification Microstructure and Melt Pool Dimensions in Electron Beam Wire Feed Additive Manufacturing of Ti-6Al4V," Additive Manufacturing, 1-4 (2014), 119-126.

8. H.E. Helmer, Carolin Körner and Robert Friedrich Singer, "Additive Manufacturing of Nickel-based Superalloy Inconel 718 by Selective Electron Beam Melting," Journal of Materials Research, 29 (2014), 1987-1996.

9. Carolin Korner et al., "Tailoring the Grain Structure of IN718 during Selective Electron Beam Melting," MATEC Web of Conferences, 14 (2014), 08001. 